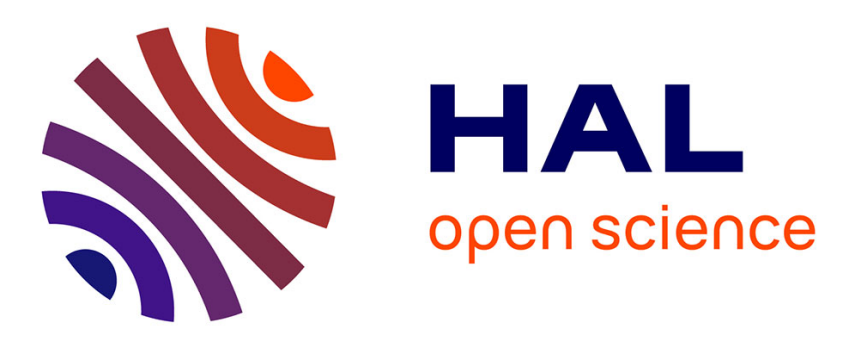

\title{
An Improved Low Rank Detector in the High Dimensional Regime
}

Pascal Vallet, Guillaume Ginolhac, Frederic Pascal, Philippe Forster

\section{To cite this version:}

Pascal Vallet, Guillaume Ginolhac, Frederic Pascal, Philippe Forster. An Improved Low Rank Detector in the High Dimensional Regime. ICASSP 2019. IEEE International Conference on Acoustics, Speech and Signal Processing (ICASSP), IEEE, May 2019, Brighton, United Kingdom. 10.1109/ICASSP.2019.8683058 . hal-02152102

\section{HAL Id: hal-02152102 https://hal.science/hal-02152102}

Submitted on 11 Jun 2019

HAL is a multi-disciplinary open access archive for the deposit and dissemination of scientific research documents, whether they are published or not. The documents may come from teaching and research institutions in France or abroad, or from public or private research centers.
L'archive ouverte pluridisciplinaire HAL, est destinée au dépôt et à la diffusion de documents scientifiques de niveau recherche, publiés ou non, émanant des établissements d'enseignement et de recherche français ou étrangers, des laboratoires publics ou privés. 


\title{
AN IMPROVED LOW RANK DETECTOR IN THE HIGH DIMENSIONAL REGIME
}

\author{
P. Vallet $^{(1)}$, G. Ginolhac $^{(2)}$, F. Pascal $^{(3)}$, P. Forster $^{(4)}$ \\ (1) IMS (CNRS, Univ. Bordeaux, Bordeaux INP), 351 cours de la Libération, 33400 Talence, France \\ (2) LISTIC (Univ. Savoie/Mont-Blanc, Polytech Annecy), 5 chemin de Bellevue, 74940 Annecy, France \\ (3) L2S (CNRS, Univ. Paris-Sud, CentraleSupélec), 3, rue Joliot-Curie, 91192 Gif sur Yvette, France \\ (4) SATIE (CNRS, Univ. Paris-Sud, ENS Paris-Saclay), 61 avenue du Président Wilson, 94235 Cachan, France \\ pascal.vallet@bordeaux-inp.fr, guillaume.ginolhac@univ-smb.fr, frederic.pascal@centralesupelec.fr, pforster@u-paris10.fr
}

\begin{abstract}
This paper introduces an improved Low Rank Adaptive Normalized Matched Filter (LR-ANMF) detector in a high dimensional (HD) context where the observation dimension is large and of the same order of magnitude than the sample size. To that end, the statistical analysis of the LR-ANMF, in a context where the target signal is disturbed by a spatially correlated Gaussian clutter and a spatially white Gaussian noise, is addressed. More specifically, the asymptotic distribution under the null hypothesis is derived, in the regime where both the dimension $M$ of the observations and the number $N$ of samples converge to infinity at the same rate and when the clutter covariance matrix has fixed rank $K$. In particular, it is shown that the LR-ANMF test statistic does not exhibit the CFAR property in the previous asymptotic regime. A correction to the LR-ANMF test is then proposed to ensure the asymptotic CFAR property, providing the improved LR-ANMF, termed as HD-LR-ANMF. Its asymptotic distribution is derived under both the null and the alternative hypotheses. Numerical simulations illustrate the fact that, despite the asymptotic nature of the analysis, the results obtained are accurate for reasonable values of $M, N$.
\end{abstract}

Index Terms - Radar target detection, Low Rank Adaptative Normalized Matched Filter, Random Matrix Theory.

\section{INTRODUCTION}

The problem of detecting a known $M$-dimensional target response corrupted by an additive disturbance with unknown spatial covariance matrix $\mathbf{R}$ is a fundamental topic in radar processing and has received a lot of attention since the past thirty years. When the disturbance is assumed to be Gaussian distributed, the problem is usually formulated as the following binary hypothesis test

$$
\mathcal{H}_{0}: \mathbf{x} \sim \mathcal{N}_{\mathbb{C}^{M}}(\mathbf{0}, \mathbf{R}) \quad \text { vs } \quad \mathcal{H}_{1}: \mathbf{x} \sim \mathcal{N}_{\mathbb{C}^{M}}(\alpha \mathbf{a}, \mathbf{R}),
$$

where $\mathbf{a} \in \mathbb{C}^{M}$ is a known signature vector and $\alpha \in \mathbb{C}$ is some unknown deterministic amplitude. In that case, the Generalized Likelihood Ratio Test (GLRT) is widely used, and consists in rejecting the null hypothesis $\mathcal{H}_{0}$ whenever the test statistic

$$
S=\frac{\left|\mathbf{a}^{*} \hat{\mathbf{R}}^{-1} \mathbf{x}\right|^{2}}{\mathbf{a}^{*} \hat{\mathbf{R}}^{-1} \mathbf{a}\left(N+\mathbf{x}^{*} \hat{\mathbf{R}}^{-1} \mathbf{x}\right)}
$$

This work has been partially supported by DGA under grant ANR-17ASTR-0015. is above a certain threshold fixed according to a desired false alarm rate, and where

$$
\hat{\mathbf{R}}=\frac{1}{N} \sum_{n=1}^{N} \mathbf{y}_{n} \mathbf{y}_{n}^{*}
$$

is the sample covariance matrix (SCM) built from $N$ i.i.d. secondary "target-free" data $\mathbf{y}_{1}, \ldots, \mathbf{y}_{N}$ distributed as $\mathcal{N}_{\mathbb{C}^{M}}(\mathbf{0}, \mathbf{R})$. The distribution of the statistic $S$ has been studied in [1] under both $\mathcal{H}_{0}$ and $\mathcal{H}_{1}$. In practice, to achieve reasonable performance in terms of detection probability, such detector requires a sample size $N$ much larger than the dimension $M$ (e.g. $N \approx 2 M$ or more in the context of array processing, see [2]).

When the covariance matrix $\mathbf{R}$ is structured according to some specific model, alternative detectors may be derived, requiring lower sample size to achieve similar detection performance than the GLRT $(1)$, see e.g. [3,4]. In this paper, we consider the scenario where the additive disturbance is the sum of a spatially white Gaussian vector modelling background noise and a spatially correlated Gaussian vector with singular covariance matrix modelling environment response (clutter):

$$
\mathbf{R}=\boldsymbol{\Gamma}+\sigma^{2} \mathbf{I},
$$

where $\sigma^{2}$ is background noise power and $\boldsymbol{\Gamma}=\sum_{k=1}^{K} \gamma_{k} \mathbf{u}_{k} \mathbf{u}_{k}^{*}$ $(K<M)$ with $\gamma_{1} \geq \ldots \geq \gamma_{K}>0$ and with $\mathbf{u}_{1}, \ldots, \mathbf{u}_{K} \in \mathbb{C}^{M}$ orthonormal. Moreover, if one has access to the spectral projection matrix $\boldsymbol{\Pi}$ onto $\operatorname{Ker}(\boldsymbol{\Gamma})$, then the clutter free observation $\tilde{\mathbf{x}}=\boldsymbol{\Pi} \mathbf{x}$ could be used with classical methods for detecting signal in white noise. In [5], assuming the clutter subspace dimension $K$ known, it was proposed to replace $\Pi$ by its Maximum Likelihood estimate $\hat{\boldsymbol{\Pi}}$, which coincides with the spectral projection matrix onto the eigenspace of $\hat{\mathbf{R}}$ associated with the $M-K$ smallest eigenvalues, and a new test statistic, termed as LR-ANMF (Low Rank Adaptive Normalized Matched Filter), was proposed:

$$
T=\frac{M\left|\mathbf{a}^{*} \hat{\boldsymbol{\Pi}} \mathbf{x}\right|^{2}}{\|\hat{\boldsymbol{\Pi}} \mathbf{a}\|_{2}^{2}\|\hat{\boldsymbol{\Pi}} \mathbf{x}\|_{2}^{2}}
$$

Other LR detectors have also been developed in the literature, see e.g. [6-8].

So far from our knowledge, the exact distribution of $T$ under both $\mathcal{H}_{0}$ and $\mathcal{H}_{1}$ remains unknown. In the large sample size regime $N \rightarrow \infty$ while $K$ and $M$ are kept fixed, $\hat{\Pi} \rightarrow \Pi$ almost surely (a.s.), and the asymptotic distribution of $M^{-1} T$ under $\mathcal{H}_{0}$ and $\mathcal{H}_{1}$ 
has been derived in [5]. In particular, under $\mathcal{H}_{0}, M^{-1} T$ is asymptotically distributed as a $\operatorname{Beta}(1, M-K-1)$ random variable, and consequently the LR-ANMF is asymptotically CFAR (Constant False Alarm Rate) in the large sample size regime. However, the theoretical results developed in the large sample size regime, which are suited for practical situations where $N \gg M$, may not be relevant when $N$ is of the same order of magnitude than $M$ (such situations occur in practice for short time stationary signals or high dimensional observations).

To model this situation, it is interesting to consider the high dimensional regime where both $M$ and $N$ converge to infinity at the same rate. Under this double asymptotic regime, $\hat{\boldsymbol{\Pi}}$ is not a consistent estimator of $\boldsymbol{\Pi}$ anymore, and the theoretical analysis of [5] is no longer valid. In this paper, we derived an improved LR-ANMF detector, termed as HD-LR-ANMF, Using classical results from random matrix theory, we provide in this paper the asymptotic distribution of $T$ under both $\mathcal{H}_{0}$ and $\mathcal{H}_{1}$ in the high dimensional regime, and under the additional assumption that the clutter $K$ is fixed with respect to $M, N$. More precisely, we prove that the LR-ANMF test statistic is no longer asymptotically CFAR in this regime. Then, providing the appropriate correction to ensure this asymptotic property leads to the new HD-LR-ANMF. The asymptotic distribution of the HD-LR-ANMF statistic is also studied under $\mathcal{H}_{1}$. Numerical simulations are provided to illustrate the fact that, despite the asymptotic nature of our analysis, the results obtained are accurate for realistic values of $M, N$.

To conclude this introduction, we also mention the approach of [9-11], where the distribution of $T$ is approximated as the distribution of the ratio of "classical" random variables (e.g. Gaussian, chi-square, Laplace, etc.), whose evaluation by Monte-Carlo methods is in practice less demanding than the evaluation of (2).

\section{SPECTRUM OF THE SCM IN THE HIGH DIMENSIONAL REGIME}

We consider hereafter the asymptotic regime in which $M=M(N)$ is a function of $N$ such that $\frac{M}{N} \rightarrow c>0$ as $N \rightarrow \infty$ and $K, \gamma_{1}, \ldots, \gamma_{K}$ are independent of $N$. We moreover assume that ${ }^{1}$ $\gamma_{1}>\ldots>\gamma_{K}>0$. We recall below some well-known results concerning the behaviour of the eigenvalues and respective orthonormal eigenvectors of the SCM $\hat{\mathbf{R}}$, which we denote by $\hat{\lambda}_{1} \geq \ldots \geq \hat{\lambda}_{M}$ and $\hat{\mathbf{u}}_{1}, \ldots, \hat{\mathbf{u}}_{M}$ in the remainder. Consider the empirical distribution of the eigenvalues of $\hat{\mathbf{R}}$ defined as the random probability measure

$$
\hat{\mu}=\frac{1}{M} \sum_{m=1}^{M} \delta_{\hat{\lambda}_{m}}
$$

As $\mathbf{R}$ is a fixed-rank perturbation of $\sigma^{2} \mathbf{I}, \hat{\mu}$ admits a.s. a weak limit as $N \rightarrow \infty$ given by the Marcenko-Pastur distribution [12,13]

$\mu(\mathrm{d} x)=(1-1 / c)^{+} \delta_{0}(\mathrm{~d} x)+\frac{\sqrt{\left(x-x^{-}\right)\left(x^{+}-x\right)}}{2 \pi \sigma^{2} c x} \mathbb{1}_{\left[x^{-}, x^{+}\right]}(x) \mathrm{d} x$

where $x^{-}=\sigma^{2}(1-\sqrt{c})^{2}, x^{+}=\sigma^{2}(1+\sqrt{c})^{2}$ and $\delta_{0}$ is the Dirac measure at 0 . As a consequence, the eigenvalues of $\hat{\mathbf{R}}$ globally spread around $\sigma^{2}$ according to the distribution $\mu$, and $\|\hat{\mathbf{R}}-\mathbf{R}\|_{2}$ does not converge to 0 anymore, as it was the case in the large sample size regime.

\footnotetext{
${ }^{1}$ The assumption that the eigenvalues of $\boldsymbol{\Gamma}$ all have multiplicity one could be relaxed and is only made here to keep light notations.
}

Concerning their individual behaviour, it is expected that the $K$ largest eigenvalues of $\hat{\mathbf{R}}$ will separate from the others when the corresponding eigenvalues $\gamma_{1}, \ldots, \gamma_{K}$ of the clutter covariance matrix $\Gamma$ are sufficiently large. The precise threshold is given in the following assumption.

Assumption 1. It holds that $\gamma_{1}>\ldots>\gamma_{K}>\sigma^{2} \sqrt{c}$.

Assumption 1 ensures that the clutter eigenvalues $\gamma_{1}, \ldots, \gamma_{K}$ are sufficiently separated from the noise eigenvalue $\sigma^{2}$, or equivalently that the clutter to noise ratio $\frac{\gamma_{k}}{\sigma^{2}}$ in eigendirection $\mathbf{u}_{k}$, for all $k=1, \ldots, K$, is roughly above $\sqrt{M / N}$. It is therefore refered to as clutter-noise separation assumption. Under Assumption 1, we have [13] that for all $k=1, \ldots, K$,

$$
\hat{\lambda}_{k} \underset{N \rightarrow \infty}{\stackrel{a . s .}{\longrightarrow}} \phi\left(\gamma_{k}, \sigma^{2}\right)=\frac{\left(\gamma_{k}+\sigma^{2}\right)\left(\gamma_{k}+\sigma^{2} c\right)}{\gamma_{k}},
$$

with $\phi\left(\gamma_{k}, \sigma^{2}\right)>x^{+}$, while

$$
\hat{\lambda}_{K+1} \underset{N \rightarrow \infty}{\stackrel{a . s}{\longrightarrow}} x^{+} \quad \text { and } \quad \hat{\lambda}_{M} \underset{N \rightarrow \infty}{\stackrel{a . s}{\longrightarrow}} x^{-}
$$

where we recall that $x^{-}$and $x^{+}$are the left and right edges of the Marcenko-Pastur distribution. Thus, under Assumption 1, the $K$ largest eigenvalues of $\hat{\mathbf{R}}$ corresponding to the clutter asymptotically split from the $M-K$ smallest eigenvalues associated to the noise.

The behaviour of the spectral projectors of $\hat{\mathbf{R}}$ associated with the $K$ largest eigenvalues has also been studied (see e.g. [14]). In particular, under Assumption 1, for any deterministic unit-norm vectors $\mathbf{b}, \mathbf{d} \in \mathbb{C}^{M}$ and all $k=1, \ldots, K$,

$$
\mathbf{b}^{*} \hat{\mathbf{u}}_{k} \hat{\mathbf{u}}_{k}^{*} \mathbf{d}=h\left(\gamma_{k}, \sigma^{2}\right) \mathbf{b}^{*} \mathbf{u}_{k} \mathbf{u}_{k}^{*} \mathbf{d}+o(1) \quad \text { a.s. }
$$

as $N \rightarrow \infty$, where $h\left(\gamma_{k}, \sigma^{2}\right)=\frac{\gamma_{k}^{2}-\sigma^{4} c}{\gamma_{k}\left(\gamma_{k}+\sigma^{2} c\right)}$. Thus, up to a factor depending only on $\gamma_{k}$ and $\sigma^{2}$, each individual entry of the projection $\hat{\mathbf{u}}_{k} \hat{\mathbf{u}}_{k}^{*}$ converges to the corresponding entry of $\mathbf{u}_{k} \mathbf{u}_{k}^{*}$.

\section{ASYMPTOTIC DISTRIBUTION OF THE LR-ANMF}

With the results of the previous section, we are now in position to study the asymptotic distribution of the statistic $T$ defined in (2) under $\mathcal{H}_{0}$ and the clutter-noise separation assumption.

The result under $\mathcal{H}_{0}$ is given as follows.

Theorem 1. Under $\mathcal{H}_{0}$ and Assumption 1,

$$
\frac{2 T}{\vartheta_{0}} \underset{N \rightarrow \infty}{\stackrel{\mathcal{D}}{\longrightarrow}} \chi^{2}(2)
$$

where $\chi^{2}(2)$ stands for the chi-square distribution with 2 degrees of freedom, and

$$
\vartheta_{0}=1+\frac{\sum_{k=1}^{K} \frac{\gamma_{k}}{\sigma^{2}}\left(1-h\left(\gamma_{k}, \sigma^{2}\right)\right)^{2}\left|\mathbf{a}^{*} \mathbf{u}_{k}\right|^{2}}{\|\mathbf{\Pi} \mathbf{a}\|_{2}^{2}+\sum_{k=1}^{K}\left(1-h\left(\gamma_{k}, \sigma^{2}\right)\right)\left|\mathbf{a}^{*} \mathbf{u}_{k}\right|^{2}} .
$$

Proof. We first focus on the denominator of (2). Using the fact that $K$ is fixed with respect to $N$, it is straightforward to show that

$$
\frac{1}{M}\|\hat{\mathbf{\Pi}} \mathbf{x}\|_{2}^{2} \underset{N \rightarrow \infty}{\stackrel{a . s .}{\longrightarrow}} \sigma^{2} .
$$


Next, using (4), we have

$$
\begin{aligned}
\|\hat{\mathbf{\Pi}} \mathbf{a}\|_{2}^{2} & =\|\mathbf{a}\|_{2}^{2}-\sum_{k=1}^{K}\left|\mathbf{a}^{*} \hat{\mathbf{u}}_{k}\right|^{2} \\
& =\|\mathbf{a}\|_{2}^{2}-\sum_{k=1}^{K} h\left(\gamma_{k}, \sigma^{2}\right)\left|\mathbf{a}^{*} \mathbf{u}_{k}\right|^{2}+o(1) \quad \text { a.s. } \\
& =\|\mathbf{\Pi} \mathbf{a}\|_{2}^{2}+\sum_{k=1}^{K}\left(1-h\left(\gamma_{k}, \sigma^{2}\right)\right)\left|\mathbf{a}^{*} \mathbf{u}_{k}\right|^{2}+o(1) \quad \text { a.s.. }
\end{aligned}
$$

We next analyze the fluctuations of the numerator of (2). Using the decomposition

$$
\mathbf{a}^{*} \hat{\boldsymbol{\Pi}} \mathbf{R} \hat{\boldsymbol{\Pi}} \mathbf{a}=\sigma^{2}\|\hat{\boldsymbol{\Pi}} \mathbf{a}\|_{2}^{2}+\sum_{k=1}^{K} \gamma_{k}\left|\mathbf{a}^{*} \hat{\boldsymbol{\Pi}} \mathbf{u}_{k}\right|^{2},
$$

as well as (6), (4), we end up with

$$
\mathbf{a}^{*} \hat{\boldsymbol{\Pi}} \hat{\mathbf{\Pi}} \mathbf{a}=\beta+o(1) \quad \text { a.s. }
$$

as $N \rightarrow \infty$, where

$$
\begin{array}{r}
\beta=\sigma^{2}\left(\|\mathbf{\Pi} \mathbf{a}\|_{2}^{2}+\sum_{k=1}^{K}\left(1-h\left(\gamma_{k}, \sigma^{2}\right)\right)\left|\mathbf{a}^{*} \mathbf{u}_{k}\right|^{2}\right) \\
+\sum_{k=1}^{K} \gamma_{k}\left(1-h\left(\gamma_{k}, \sigma^{2}\right)\right)^{2}\left|\mathbf{a}^{*} \mathbf{u}_{k}\right|^{2}
\end{array}
$$

Since for all $u \in \mathbb{R}$ and $\xi \in \mathbb{C}$, we have

$$
\mathbb{E}\left[\exp \left(\mathrm{i} u \operatorname{Re}\left(\xi \mathbf{a}^{*} \hat{\boldsymbol{\Pi}} \mathbf{x}\right)\right)\right]=\mathbb{E}\left[\exp \left(-u^{2}|\xi|^{2} \mathbf{a}^{*} \hat{\mathbf{\Pi}} \mathbf{R} \hat{\boldsymbol{\Pi}} \mathbf{a} / 4\right)\right],
$$

using (7) and dominated convergence theorem together with $\xi=$ $\sqrt{2 / \beta}$ gives

$$
\mathbb{E}\left[\exp \left(\operatorname{i} u \operatorname{Re}\left(\xi \mathbf{a}^{*} \hat{\boldsymbol{\Pi}} \mathbf{x}\right)\right)\right]=\exp \left(-u^{2} / 2\right)+o(1)
$$

as $N \rightarrow \infty$. Consequently, Levy's theorem implies that

$$
\frac{2}{\beta}\left|\mathbf{a}^{*} \hat{\boldsymbol{\Pi}} \mathbf{x}\right|^{2} \underset{N \rightarrow \infty}{\stackrel{\mathcal{D}}{\longrightarrow}} \chi^{2}(2),
$$

and the results of Theorem 1 follows from (5), (6) and (9).

As a first consequence of Theorem 1, we notice that when $M, N$ are sufficiently large such that $\frac{M}{N} \approx 0$, the statistic $2 T$ is approximately distributed as a $\chi^{2}(2)$ random variable, which is consistent with the results in the large sample regime obtained in [5] (since in terms of distribution, $2 \operatorname{Beta}(1, M-K) \rightarrow \chi^{2}(2)$ as $\left.M \rightarrow \infty\right)$. Second, when the target signature is nearly orthogonal to the clutter subspace, that is $\Pi \mathbf{a} \approx \mathbf{a}$, then $2 T$ is also approximately distributed as $\chi^{2}(2)$ random variable. Third, when the clutter-to-noise ratio is large (i.e. $\gamma_{1}, \ldots, \gamma_{K} \gg \sigma^{2}$ ), then the same conclusion is reached. Thus, in the three previous scenarios, the test statistic $T$ has approximately the CFAR property.

The previous remarks shed light on a situation where the distribution of $T$ under $\mathcal{H}_{0}$ will critically depend on the clutter statistical properties. Indeed, this situation will occur when $M \approx N$ and $\Pi$ a is not close to $\mathbf{a}$, that is in the high dimensional regime and when the target signature projects a non-vanishing energy onto the clutter subspace. Thus, in this context, $T$ is not asymptotically CFAR, and using the chi-square or beta distributions to set the test threshold according to a desired false alarm rate may cause serious errors in the performance prediction. We propose in the next section an improved version of the LR-ANMF to correct this main drawback and obtain a LR detector with the CFAR property.

\section{IMPROVED VERSION OF THE LR-ANMF}

As we have seen from Theorem 1, the asymptotic distribution of $T$ under the null hypothesis $\mathcal{H}_{0}$ depends on the clutter covariance matrix $\Gamma$ and the noise power $\sigma^{2}$, which are not available in practice. We propose in this section to modify the test statistic $T$ so that its asymptotic distribution under $\mathcal{H}_{0}$ no longer depends on these parameters. The new test statistic is defined as follows. Let

$$
\hat{\sigma}^{2}=\frac{1}{M-K} \sum_{k=K+1}^{M} \hat{\lambda}_{k}
$$

be the usual ML estimate of $\sigma^{2}$, and denote by $\hat{\gamma}_{k}$ the unique solution to the equation $\phi\left(\gamma, \hat{\sigma}^{2}\right)=\hat{\lambda}_{k}$ in the interval $\left(\hat{\sigma}^{2} \sqrt{c},+\infty\right)$, when it exists, and $\hat{\gamma}_{k}=\hat{\sigma}^{2} \sqrt{c}$ otherwise.

Definition 1 (High-Dimensional Low-Rank Adaptive Normalized Matched Filter - HD-LR-ANMF).

$$
\tilde{T}=\frac{2\left|\mathbf{a}^{*} \hat{\boldsymbol{\Pi}} \mathbf{x}\right|^{2}}{\tilde{\vartheta}_{0}},
$$

where $\tilde{\vartheta}_{0}=\hat{\sigma}^{2}\|\hat{\mathbf{\Pi}} \mathbf{a}\|_{2}^{2}+\sum_{k=1}^{K} \hat{\gamma}_{k} \frac{\left(1-h\left(\hat{\gamma}_{k}, \hat{\sigma}^{2}\right)\right)^{2}}{h\left(\hat{\gamma}_{k}, \hat{\sigma}^{2}\right)}\left|\mathbf{a}^{*} \hat{\mathbf{u}}_{k}\right|^{2}$.

We then have the following result.

Theorem 2. Under $\mathcal{H}_{0}$ and Assumption 1,

$$
\tilde{T} \underset{N \rightarrow \infty}{\stackrel{\mathcal{D}}{\longrightarrow}} \chi^{2}(2) .
$$

Proof. From the convergence (9), we only have to estimate consistently the term $\beta$ defined in (8). Using (6) and the fact that $\hat{\sigma}^{2} \rightarrow \sigma^{2}$ a.s. as $N \rightarrow \infty$, we have

$$
\begin{aligned}
\hat{\sigma}^{2}\|\hat{\mathbf{\Pi}} \mathbf{a}\|_{2}^{2} & = \\
& \sigma^{2}\left(\|\mathbf{\Pi} \mathbf{a}\|_{2}^{2}+\sum_{k=1}^{K}\left(1-h\left(\gamma_{k}, \sigma^{2}\right)\right)\left|\mathbf{a}^{*} \mathbf{u}_{k}\right|^{2}\right)+o(1) \quad \text { a.s. }
\end{aligned}
$$

From Assumption 1 and (3), for all $k \in\{1, \ldots, K\}, \hat{\lambda}_{k} \in$ $\left(\hat{\sigma}^{2}(1+\sqrt{c})^{2},+\infty\right)$ a.s. for all large $N$, and since $\gamma \mapsto \phi\left(\gamma, \hat{\sigma}^{2}\right)$ is an increasing one-to-one mapping from $\left(\hat{\sigma}^{2} \sqrt{c},+\infty\right)$ onto $\left(\hat{\sigma}^{2}(1+\sqrt{c})^{2},+\infty\right)$, the equation $\phi\left(\gamma, \hat{\sigma}^{2}\right)=\hat{\lambda}_{k}$ admits a unique solution $\hat{\gamma}_{k}>\hat{\sigma}^{2} \sqrt{c}$. By continuity and convergences $\hat{\sigma}^{2} \rightarrow \sigma^{2}$ and $\hat{\lambda}_{k} \rightarrow \phi\left(\gamma_{k}, \sigma^{2}\right)$, we deduce $\hat{\gamma}_{k} \rightarrow \gamma_{k}$ a.s as $N \rightarrow \infty$. Finally, from (4), we obtain

$$
\begin{aligned}
& \sum_{k=1}^{K} \gamma_{k}\left(1-h\left(\gamma_{k}, \sigma^{2}\right)\right)^{2}\left|\mathbf{a}^{*} \mathbf{u}_{k}\right|^{2}= \\
& \quad \sum_{k=1}^{K} \frac{\hat{\gamma}_{k}\left(1-h\left(\hat{\gamma}_{k}, \hat{\sigma}^{2}\right)\right)^{2}}{h\left(\hat{\gamma}_{k}, \hat{\sigma}^{2}\right)}\left|\mathbf{a}^{*} \hat{\mathbf{u}}_{k}\right|^{2}+o(1) \quad \text { a.s. }
\end{aligned}
$$

which implies $\tilde{\vartheta}_{0}=\beta+o(1)$ a.s., and the result of Theorem 2 . 
To conclude this section, we also provide the asymptotic distribution of the HD-LR-ANMF $\tilde{T}$ under $\mathcal{H}_{1}$ in the next theorem. The proof, which is very similar to the proof of Theorem 1 and Theorem 2 , is omitted due to space constraints.

Theorem 3. Under $\mathcal{H}_{1}$ and Assumption 1,

$$
\sup _{x \in \mathbb{R}}\left|\mathbb{P}(\tilde{T} \leq x)-F\left(x ; 2, \frac{2|\alpha|^{2}}{\sigma^{2} \vartheta_{1}}\right)\right| \underset{N \rightarrow \infty}{\longrightarrow} 0,
$$

where $x \mapsto F(x ; k, \Delta)$ denotes the cumulative distribution of the non-central chi-square distribution with $k$ degrees of freedom and non-centrality parameter $\Delta$, and where

$$
\vartheta_{1}=\frac{\vartheta_{0}}{\|\mathbf{\Pi} \mathbf{a}\|_{2}^{2}+\sum_{k=1}^{K}\left(1-h\left(\gamma_{k}, \sigma^{2}\right)\right)\left|\mathbf{a}^{*} \mathbf{u}_{k}\right|^{2}} .
$$

\section{NUMERICAL RESULTS}

In this section, we provide numerical examples illustrating the results of the previous section, in the context of array processing where $M$ represents the number of sensors in a uniform linear array with spacing of half the wavelength, which implies that the signature vector is further parametrized as

$$
\mathbf{a}(\theta)=\frac{1}{\sqrt{M}}\left(1, \mathrm{e}^{-\mathrm{i} \pi \sin (\theta)}, \ldots, \mathrm{e}^{-\mathrm{i}(M-1) \pi \sin (\theta)}\right)^{T},
$$

where $\theta \in\left[-\frac{\pi}{2}, \frac{\pi}{2}\right]$ represents the Direction of Arrival (DoA). The clutter is composed of $K=3$ sources with DoA $-20^{\circ}, 0^{\circ}$ and $20^{\circ}$ and the target has DoA $20.1^{\circ}$. The clutter is renormalized to have unit energy after matched filtering with target signature. The clutter to noise ratio (CNR) and signal to noise ratio (SNR) after matched filter (CNR) are defined respectively as $\frac{1}{\sigma^{2}}$ and $\frac{|\alpha|^{2}}{1+\sigma^{2}}$. Empirical CDFs and Pfa are evaluated with $10^{4}$ trials.

In Figures 1 and 2, we plot the empirical CDFs of $\tilde{T}$ for different values of $M=N$ as well as the asymptotic limits (CDF of the $\chi^{2}(2)$ distribution under $\mathcal{H}_{0}$ and CDF of the $\chi^{2}\left(2, \frac{2|\alpha|^{2}}{\sigma^{2} \vartheta_{1}}\right)$ distribution under $\mathcal{H}_{1}$ ). As expected, we notice that the empirical plots of $\tilde{T}$ get closer to the asymptotic limits when the data size grows.

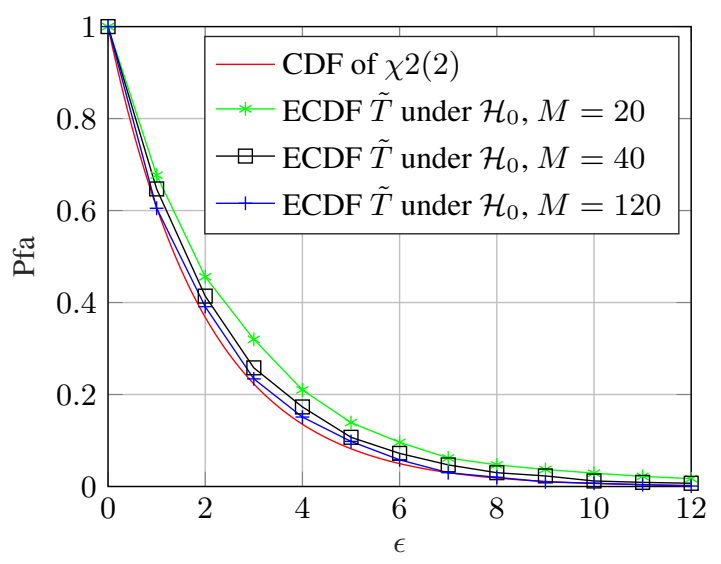

Fig. 1. ECDFs of $\tilde{T}$ under $\mathcal{H}_{0}$ hypothesis for different values of $M$ and $\mathrm{CDF}$ of $\chi^{2}(2)$ (red). $N=M, K=3, C N R=10 \mathrm{~dB}$.

In Figure 3, we show the empirical Pfa as a function of the target DoA for $\frac{2 T}{\vartheta_{0}}, T$ and $\tilde{T}$. We notice that the correction $\frac{2}{\vartheta_{0}}$ on $T$ allows

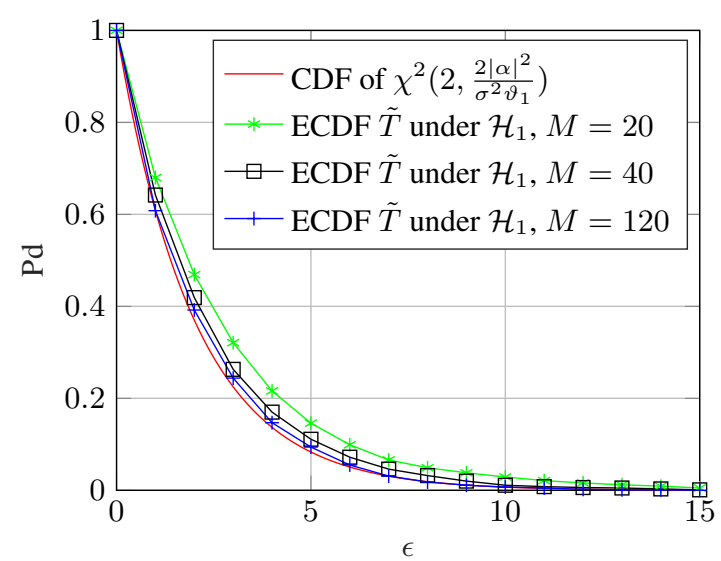

Fig. 2. ECDFs of $\tilde{T}$ under $\mathcal{H}_{1}$ hypothesis for different values of $M$ and CDF of $\chi^{2}\left(2, \frac{2|\alpha|^{2}}{\sigma^{2} \vartheta_{1}}\right)$ (red). $N=M, K=3, C N R=10 d B$, $S N R=-20 d B$.

to obtain a CFAR detector and that $\tilde{T}$ is a correct approximation of $\frac{2 T}{\vartheta_{0}}$ for a standard data size in many applications like STAP or MIMO RADAR.

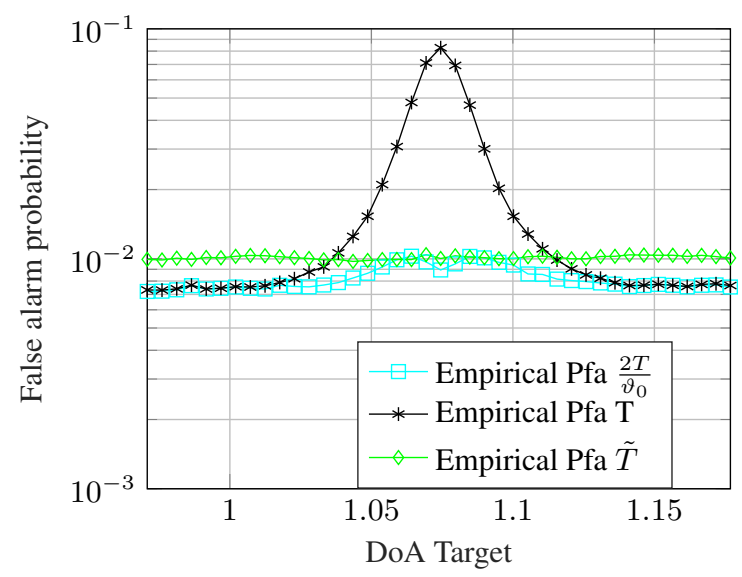

Fig. 3. Empirical Pfa as a function of the DoA for $\frac{2 T}{\vartheta_{0}}, T$ and $\tilde{T}$. $M=80, N=80, K=3, C N R=10 d B$.

\section{CONCLUSION}

In this paper, we have addressed the study of the LR-ANMF test statistic for target detection. Considering the asymptotic regime in which the dimension of the observations and the number of secondary samples converge to infinity at the same rate, we have proved that the test statistic, suitably renormalized by a factor depending on the noise and clutter parameters, is asymptotically $\chi^{2}$-distributed under the null hypothesis. Based on this result, we have proposed a new test, the HD-LR-ANMF, possessing the asymptotic CFAR property. Numerical simulations have been provided which indicate that our results are accurate for realistic values of sample size and observations dimension. 


\section{REFERENCES}

[1] E.J. Kelly, “An adaptive detection algorithm," IEEE Trans. Aerosp. Electron. Syst., , no. 2, pp. 115-127, 1986.

[2] R. Nitzberg, "Detection loss of the sample matrix inversion technique," IEEE Trans. Aerosp. Electron. Syst., , no. 6, pp. 824-827, 1984.

[3] A. Haimovich, "Asymptotic distribution of the conditional signal-to-noise ratio in an eigenanalysis-based adaptive array," IEEE Trans. on Aero. and Elec. Syst., vol. 33, pp. 988 - 997, 1997.

[4] G. Ginolhac, P. Forster, F. Pascal, and J.P. Ovarlez, "Performance of two low-rank STAP filters in a heterogeneous noise," IEEE Trans. Signal Process., vol. 61, no. 1, pp. 57 - 61, January 2013.

[5] M. Rangaswamy, F.C. Lin, and K.R. Gerlach, "Robust adaptive signal processing methods for heterogeneous radar clutter scenarios," Signal Process., vol. 84, no. 9, pp. 1653-1665, 2004.

[6] A. Dogandzic and B. Zhang, "Complex signal amplitude estimation and adaptive detection in unknown low-rank interference," in Proceedings of Asilomar Conference, Pacific Grove, CA, USA, November 2006.

[7] A. Dogandzic and B. Zhang, "Bayesian complex amplitude estimation and adaptive matched filter detection in low-rank interference," IEEE Trans. Signal Process., vol. 55, no. 3, pp. 1176 - 1182, march 2007.

[8] J.-F. Degurse, L. Savy, and S. Marcos, "Reduced-rank STAP for target detection in heterogeneous environments," IEEE Trans. on Aero. and Elec. Syst., vol. 50, no. 2, pp. 1153 - 1162, april 2014.

[9] A. Combernoux, F. Pascal, G. Ginolhac, and M. Lesturgie, "Asymptotic performance of the Low Rank Adaptive Normalized Matched Filter in a large dimensional regime," in IEEE International Conference on Acoustics, Speech and Signal Processing (ICASSP). IEEE, 2015, pp. 2599-2603.

[10] G. Ginolhac and P. Forster, "Approximate distribution of the low-rank adaptive normalized matched filter test statistic under the null hypothesis," IEEE Trans. Aerosp. Electron. Syst., vol. 52, no. 4, 2016.

[11] A. Combernoux, F. Pascal, and G. Ginolhac, "Performance of the Low Rank Adaptive Normalized Matched Filter test under a large dimension regime," IEEE Trans. Aerosp. Electron. Syst., 2018.

[12] V.A. Marcenko and L.A. Pastur, "Distribution of eigenvalues for some sets of random matrices," Mathematics of the USSRSbornik, vol. 1, pp. 457, 1967.

[13] J. Baik, G. Ben Arous, and S. Péché, "Phase transition of the largest eigenvalue for nonnull complex sample covariance matrices," Ann. Probab., vol. 33, no. 5, pp. 1643-1697, 2005.

[14] Florent Benaych-Georges and Raj Rao Nadakuditi, "The eigenvalues and eigenvectors of finite, low rank perturbations of large random matrices," Advances in Mathematics, vol. 227, no. 1, pp. 494-521, 2011. 